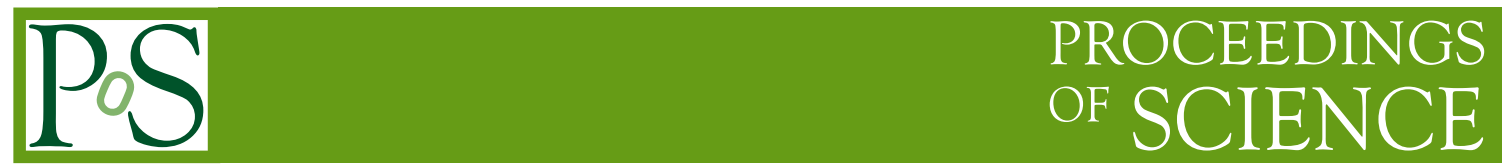

\title{
Measurements of ttbar+X using the ATLAS detector
}

\section{Yichen Li*}

Siegen University

E-mail: yichen.li@cern.ch

On behalf of the ATLAS collaboration

The large integrated luminosity provided by the LHC enables the production of a significant number of top-quark pairs in association with additional jets or additional gauge bosons. Analysis for all of these final states are presented here. The measurement of the production of top-quark pairs in association with $\mathrm{W}$ and $\mathrm{Z}$ bosons uses events with multiple leptons and in particular probes the coupling between the top quark and the $\mathrm{Z}$ boson. The production of top-quark pairs in association with additional light or heavy jets is an important background to searches for new physics.

38th International Conference on High Energy Physics 3-10 August 2016

Chicago, USA

\footnotetext{
*Speaker.
} 


\section{Introduction}

With the high center of mass energy $(\sqrt{s})$ and large integrated luminosity of the LHC, the associated production of additional gauge bosons $(W / Z / \gamma)$ together with top anti-top quark pair $(t \bar{t})$, known as $t \bar{t} V$ production, can be studied. Among it, the $t \bar{t} Z$ and $t \bar{t} \gamma$ production are a direct probe of the couplings between the top quark and $Z / \gamma$ bosons, which have never been directly measured in previous collider experiments and can be modified in some Beyond Standard Model (BSM) theories so that they are sensitive to new physics. As for $t \bar{t} W$, it has similar signature as many new physics thus is an important background requiring careful study.

The $t \bar{t}$ production with additional QCD radiation (gluon/quark), especially of heavy flavour, is another type of event that is interesting to investigate at the LHC. Not only because its measurement is a test of our understanding of QCD at an ever high energy scale pushed by the LHC, but also that it is a primary source of background to many new physics searches. Besides, on the technical side, this kind of measurement can be used for tuning Monte Carlo (MC) generators on the market.

In this proceeding, the measurements made with the ATLAS detector [1] of these $t \bar{t}$ associated productions, which are collectively referred to as $t \bar{t}+X$, will be presented using the proton-proton $(p p)$ collision data collected by the ATLAS detector at center of mass energies between 7 and 13 $\mathrm{TeV}$.

\section{2. $t \bar{t}+W / Z$ production}

The associated production of $t \bar{t} W$ and $t \bar{t} Z$ are measured together based on an analysis of 20.3 $\mathrm{fb}^{-1}$ data collected at $\sqrt{s}=8 \mathrm{TeV}$ in year 2012 [2]. Examples of their leading-order (LO) Feynman diagrams at the LHC are shown in Figure 1.

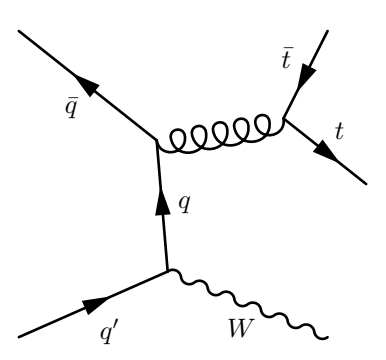

(a)

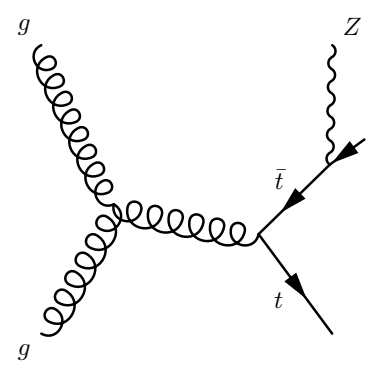

(b)

Figure 1: Example leading-order Feynman diagrams for (a) $t \bar{t} W$ and (b) $t \bar{t} Z$ production.

Depending on the decay mode of the $t \bar{t}$ pair and the W/Z boson, there will be zero to four prompt leptons in the final state, among which those with two/three/four leptons are used and are called dilepton( $2 \mathrm{~L}$ )/trilepton(3L)/tetralepton( $4 \mathrm{~L}$ ) channel. The $2 \mathrm{~L}$ channel is further divided into opposite-sign dilepton (2LOS) and same-sign dilepton (2LSS) channels according to the charges of the two leptons. The $2 \mathrm{LOS}, 3 \mathrm{~L}$ and $4 \mathrm{~L}$ channels are mostly sensitive to $t \bar{t} Z$ production while the 2LSS channel is dedicated for $t \bar{t} W$ production. Besides, each of these channels further splits into several signal regions (SR), as shown in Figure 2, either to disentangle the $t \bar{t} W$ and $t \bar{t} Z$ signals or to do separate optimization to enhance signal sensitivity. 
In the 2LOS channel, the main background comes from $t \bar{t}$ and $Z$ production, both of which are constrained with control regions (CR). In the 2LSS channel, the instrumental backgrounds, which are the fake leptons and charge misidentified leptons, dominate and they are estimated with datadriven methods. In the $3 \mathrm{~L}$ and $4 \mathrm{~L}$ channels where there are high signal over background ratios, the leading background is diboson (VV) production, which is also constrained in CRs. Besides, there are some small Standard Model (SM) backgrounds, like $t \bar{t} H, t Z$ and $W t Z$, across each channels and they are categorized as "rare SM". Figure 2 illustrates the background compositions and shows data/MC comparisons in each SRs and CRs.
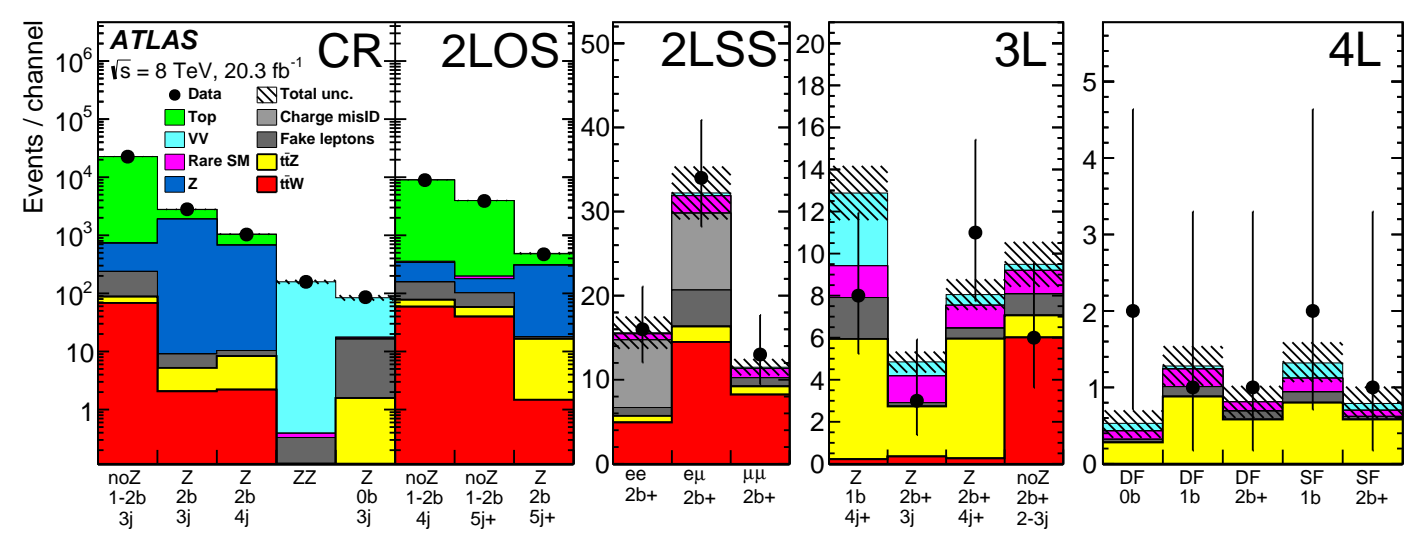

Figure 2: Post-fit expected event yields compared to data in the SRs and CRs. The hatched area corresponds to the total uncertainty on the predicted yields [2].

The cross sections for the $t \bar{t} W$ and $t \bar{t} Z$ production are simultaneously fitted to be $369_{-79}^{+86}$ (stat.) \pm 44 (syst.) fb and $176_{-48}^{+52}$ (stat.) \pm 24 (syst.) fb respectively, agreeing well with their corresponding next-to-leading order (NLO) calculations of $232 \pm 32 \mathrm{fb}$ and $215 \pm 30 \mathrm{fb}$ from MADGRAPH5_AMC @ NLO (MG5_aMC) [4]. The comparison is illustrated in Figure 3a. The statistical uncertainty dominates the measurement and the leading systematic uncertainty comes from background estimation.

The observed (expected) significances of the $t \bar{t} W$ and $t \bar{t} Z$ signal are quantified to be $5.0 \sigma$ $(3.2 \sigma)$ and $4.2 \sigma(4.5 \sigma)$ respectively over the signal-free model, which establishes the observation of the $t \bar{t} W$ production and reveals evidence of the $t \bar{t} Z$ production. Besides, the background-only hypothesis with neither $t \bar{t} W$ and $t \bar{t} Z$ is excluded with an observed (expected) significance of 7.1 $\sigma$ $(5.9 \sigma)$.

The same analysis is performed using $3.2 \mathrm{fb}^{-1}$ data collected at $\sqrt{s}=13 \mathrm{TeV}$ in the year 2015 in the $2 \mathrm{LSS}, 3 \mathrm{~L}$ and $4 \mathrm{~L}$ channels [3]. The measured cross sections are $1.50 \pm 0.72$ (stat.) \pm 0.33 (syst.) $\mathrm{pb}$ and $0.92 \pm 0.29$ (stat.) \pm 0.10 (syst.) $\mathrm{pb}$ for the $t \bar{t} W$ and $t \bar{t} Z$ respectively. They agree with their NLO predictions of $0.60 \pm 0.08 \mathrm{pb}$ and $0.84 \pm 0.09 \mathrm{pb}$ from MG5_aMC within the uncertainty. The comparison is shown in Figure $3 b$.

\section{3. $t \bar{t}+\gamma$ production}

The associated production of $t \bar{t} \gamma$ is measured with $4.6 \mathrm{fb}^{-1}$ of data collected in the year 2011 at $\sqrt{s}=7 \mathrm{TeV}$ [5]. The analysis is performed in the single lepton channel with events containing one 


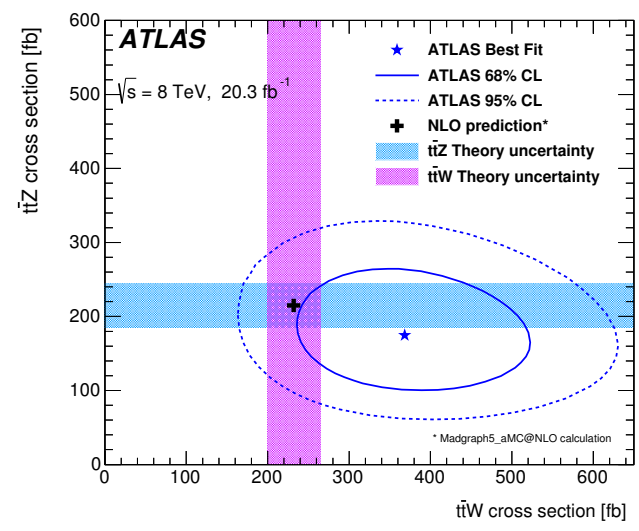

(a)

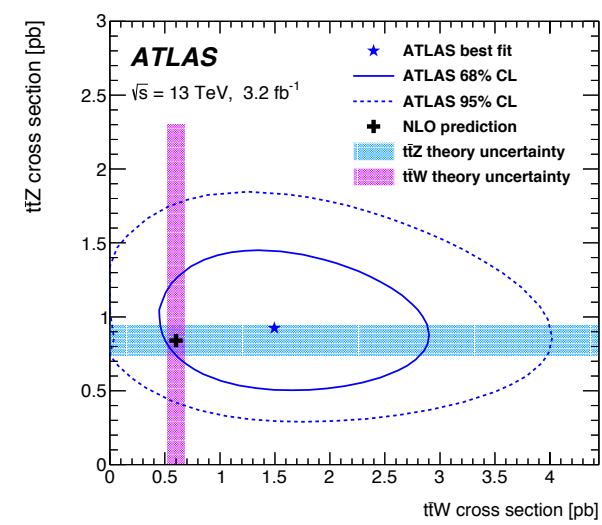

(b)

Figure 3: The simultaneously fitted $t \bar{t} W$ and $t \bar{t} Z$ cross sections using (a) $8 \mathrm{TeV}$ data [2] and (b) 13 $\mathrm{TeV}$ data [3], along with the $68 \%$ and $95 \%$ confidence level contours. The Shaded areas correspond to the theoretical uncertainties in the SM predictions.

photon. The photon is required not to be too close to the lepton $(\Delta R>0.7)$ or jets $(\Delta R>0.5)$ in the event to suppress photon radiation from $t \bar{t}$ decay products, which are not related to the top-photon coupling.

The main background comes from events that have a fake photon, whose origin is either a jet (hadron fake) or an electron ( $e \rightarrow \gamma$ fake). The hadron fake is extracted together with the cross section in a template fit, where the template is constructed using a photon isolation variable. The $e \rightarrow \gamma$ fake contribution is extrapolated from a $t \bar{t}+$ electron control region using $e \rightarrow \gamma$ fake rate, which is derived from $Z \rightarrow e e$ events with one of the electron being mis-reconstructed as a photon. Other small backgrounds come from SM processes accompanied with photon radiation.

The cross section for the $t \bar{t} \gamma$ production and its subsequent single leptonic decay is measured in a fiducial region to be $63 \pm 8$ (stat.) ${ }_{-13}^{+17}$ (syst.) \pm 1 (lumi.) fb, which agrees with the prediction of 48 (47) $\pm 10 \mathrm{fb}$ from WHIZARD [7] (MadGraph [8]) normalized to NLO precision using a $\mathrm{k}$-factor [6]. The systematics is mainly from the uncertainty of jet modelling.

The observed signal significance over the background-only model is quantified to be $5.3 \sigma$, which is the first direct observation of the $t \bar{t} \gamma$ production.

\section{4. $t \bar{t}+(b)$ jets production}

The activities of additional jets produced in association with $t \bar{t}$, which are to be distinguished from jets produced by the $t \bar{t}$ decay, are studied with both the $8 \mathrm{TeV}$ and $13 \mathrm{TeV}$ data.

The $8 \mathrm{TeV} t \bar{t}+$ jets measurement is performed in the $e \mu$ channel [9]. Multiplicity and transverse momentum $\left(p_{T}\right)$ of the additional jets are measured, as well as the gap fraction which is the fraction of $t \bar{t}$ events without additional QCD radiation in a certain gap of the detector acceptance. Figure 4 shows the unfolded $p_{T}$ distribution of the leading additional jet and multiplicity distribution of jets with $p_{T}$ larger than $25 \mathrm{GeV}$, compared to predictions from various MC generators with different 
tunes. Good agreements between the unfolded results and MC predictions are observed for most NLO generators and some LO generators with a particular tune.

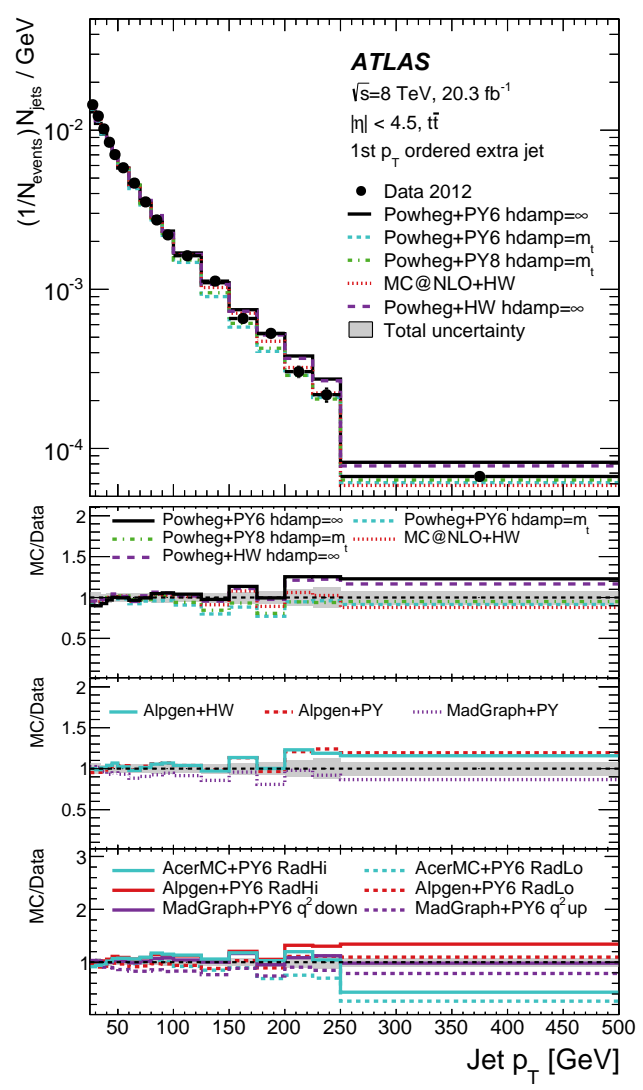

(a)

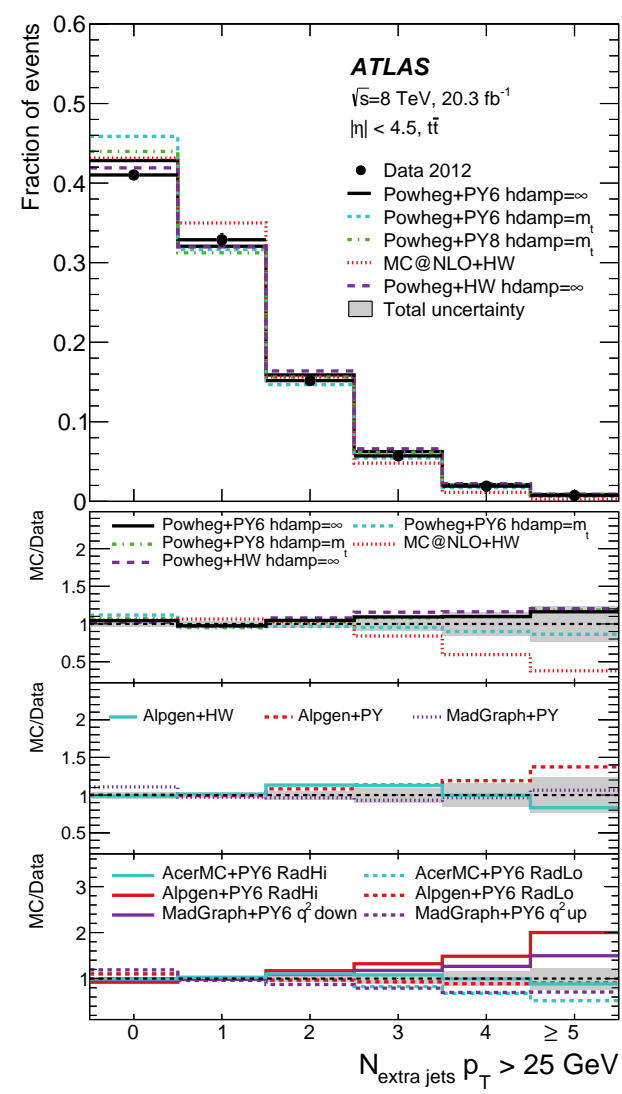

(b)

Figure 4: Comparison between measurements and predictions from NLO and LO generators for (a) the $p_{T}$ spectrum of the leading extra jet and (b) the jet multiplicity with $p_{T}>25 \mathrm{GeV}$ [9].

The $13 \mathrm{TeV} t \bar{t}+$ jets measurement is performed in all the dilepton channels [10], focusing on the normalized additional jet multiplicity distributions and on the comparison with several NLO generators, where again good agreement is observed.

The additional heavy flavour QCD radiation associated with $t \bar{t}$ production is studied with the $8 \mathrm{TeV}$ data, by performing fiducial cross section measurement for the $t \bar{t} b$ and $t \bar{t} b b$ events in the leptonic channels [11]. The results are compared with predictions from NLO and LO generators and in general good agreements are achieved as shown in Figure 5, except for some extreme $g \rightarrow b \bar{b}$ splitting models in Pythia8 [12].

\section{Conclusion}

ATLAS has established the existence of $t \bar{t} W$ production and shown evidence of the $t \bar{t} Z$ production with its $8 \mathrm{TeV}$ data. The two productions are also measured with the early $13 \mathrm{TeV}$ data and results show good agreement with theoretical predictions. The other $t \bar{t} V$ production, which is 


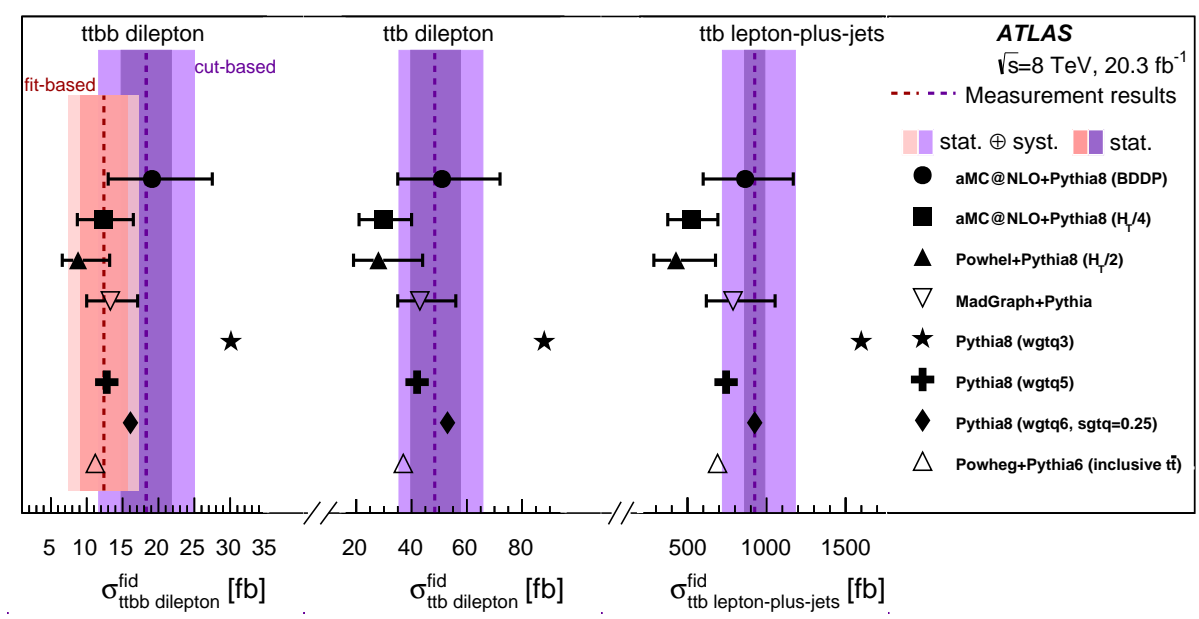

(a)

Figure 5: Comparison of the measured fiducial cross sections with theoretical predictions obtained from a variety of different generators. The coloured bands indicate the statistical and total uncertainties of the measurements [11].

the $t \bar{t} \gamma$, is observed with an analysis of the $7 \mathrm{TeV}$ data. Besides, $t \bar{t}$ plus additional (heavy flavour) QCD radiation is well measured in $8 \mathrm{TeV}$ and $13 \mathrm{TeV}$ data. In general, NLO and LO generators give predictions that agree with the measurement results. These results will be useful for tuning the MC generators in the future.

\section{References}

[1] ATLAS Collaboration, JINST 3 (2008) S08003.

[2] ATLAS Collaboration, JHEP 11 (2015) 172.

[3] ATLAS Collaboration, arXiv:1609.01599 [hep-ex].

[4] J. Alwall et al., JHEP 07 (2014) 079.

[5] ATLAS Collaboration, PRD 91 (2015) 072007.

[6] K. Melnikov, A. Scharf, and M. Schulze, PRD 83 (2011) 074013.

[7] W. Kilian, T. Ohl, and J. Reuter, EPJC 71 (2011) 1742.

[8] F. Maltoni and T. Stelzer, JHEP 02 (2003) 027.

[9] ATLAS Collaboration, JHEP 09 (2016) 074

[10] ATLAS Collaboration, ATLAS-CONF-2015-065, http://cds.cern.ch/record/2114832.

[11] ATLAS Collaboration, EPJC 76 (2016) 11.

[12] T. Sjöstrand et al., Comput. Phys. Commun. 191 (2015) 159-177. 